\title{
Modeling and Simulation of a Ship Launched Glider Cargo Delivery System
}

\author{
Anand Puranik ${ }^{*}$, Gordon Parker ${ }^{\dagger}$ and Chris Passerello \\ Michigan Technological University, Houghton, MI 49931-1295 \\ J. Dexter Bird ${ }^{\S}$, III \\ Craft Engineering Associates, Inc., Hampton, VA 23661-1202 \\ Oleg Yakimenko" and Isaac Kaminer \\ Naval Postgraduate School, Monterey, CA 93943-5146
}

\begin{abstract}
The paper deals with the high-fidelity modeling and simulation of a powered parafoil-payload system with respect to its application in autonomous precision airborne cargo delivery. In the proposed concept the cargo transfer is accomplished in two phases: Initial towing phase when the glider follows the towing vessel in a passive lift mode and the autonomous gliding phase when the system is guided to the desired point. During the towing phase, the system gains as much altitude as possible by taking the angle-of-attack that will provide the best lift. Once sufficient altitude is attained, the gliding phase starts. The system is steered to the desired location by controlling the lengths of the rear suspension lines using two control inputs. The paper presents the concept of the system, its $6 \mathrm{DoF}$ model, the control algorithm at the stage of passive glide and the simulation results.
\end{abstract}

\section{Introduction}

T $\mathrm{N}$ the past, the modeling of the parafoil-payload system has been done with respect to various applications such as 1 NASA assured crew return vehicle, payload recovery in sounding rocket flights, supply troops with ammunition and other essentials from airplane, etc. In all these applications the payload along with the parafoil is released from a certain height and then it is guided to the pre-defined target point on the earth. During littoral operations there is a demand to supply troops. It is required that the system is capable of delivering goods from the ship onto unprepared spots on the shore, be passive and quiet.

Figure 1 represents the underlying concept of the proposed system. The $550 \mathrm{sq} \mathrm{ft}$ double-skin parafoil with $750 \mathrm{lb}$ payload is being towed and released at the altitude of about $2000-2500$ feet. It then gains as much altitude as possible in a powered mode and then glides shoreward. It is assumed that the airborne autopilot will use way-point navigation to deliver supplies to the predetermined position within the range of a couple of miles. Close to the target it performs a wind-determining maneuver and safely lands into the wind.

This paper describes current status of the project, developed parafoil-payload system model, mathematical model of the system and results of simulations. Along with the analysis of passive glide, the paper focuses on the influence of the powered flight on the dynamics of the system. The paper also addresses the environmental effects on the dynamics of the flight such as changes in the wind velocity and the density of air. It finally summarizes the observations and conclusions from the typical simulation results.

\footnotetext{
* Graduate Student, Mechanical Engineering - Engineering Mechanics Department, 1400 Townsend Drive, aspurani@mtu.edu, Student Member AIAA.

$\dagger$ Professor, Mechanical Engineering - Engineering Mechanics Department, 1400 Townsend Drive, cepass@mtu.edu, AIAA Member.

* Professor, Mechanical Engineering - Engineering Mechanics Department, 1400 Townsend Drive, ggparker@mtu.edu,AIAA Member.

$\S$ Senior Engineer, $210248^{\text {th }}$ Street.

ๆ Research Associate Professor, Department of Mechanical and Astronautical Engineering, Code MAE/Yk, oayakime@nps.edu, AIAA Associate Fellow.

\# Professor, Department of Mechanical and Astronautical Engineering, Code MAE/Ka, kaminer@nps.edu, AIAA Senior Member.
} 

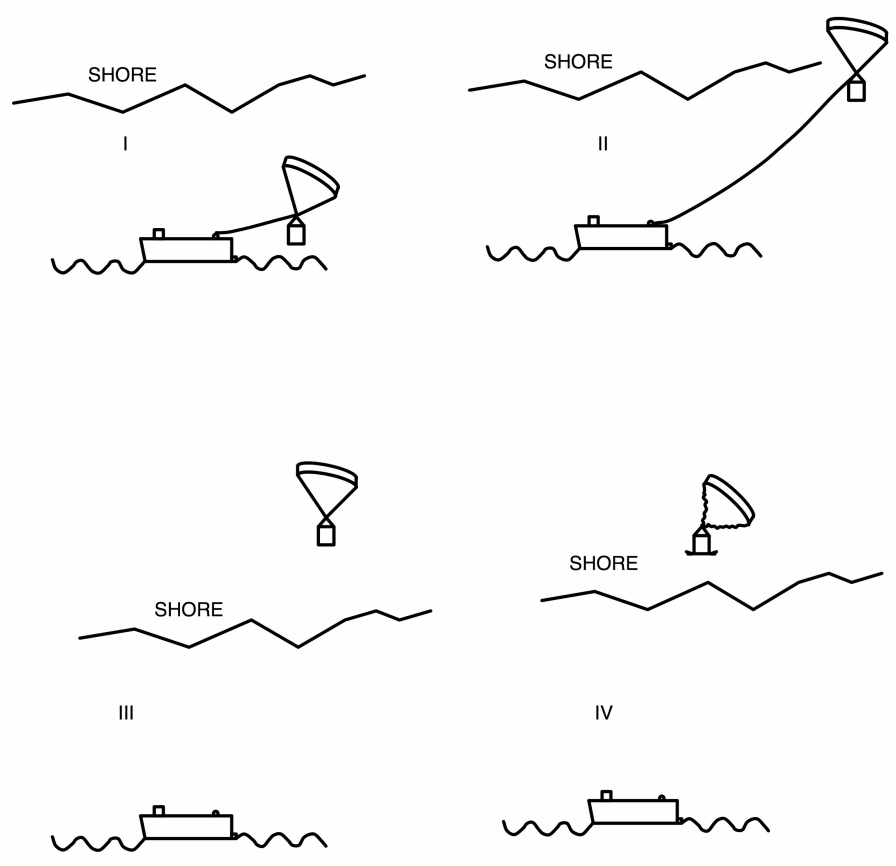

Figure 1. Stages of deployment

\section{The Description of the System}

Figure 2 shows a schematic of the parasail-payload system labeled with the 4 major points used in the equation of motion development. The tow cable emanates from a location near the stern of the ship at point 1 and is attached to the parasail system at point 3 . The tow line length $L(t)$, and its first two derivatives, $\dot{L}(t)$ and $\ddot{L}(t)$, are assumed to be known. The payload and the parasail are assumed to consist of a single, 6 degree of freedom rigid body with mass $m$ and inertia matrix $\mathbf{I}$. Its center of mass is at point 2 . The center of pressure, where the lift and drag forces are assumed to act, is at point 4 . Point 5 denotes the location of the constant thrust engine. The inertial frame is also depicted in Figure 1 and will be abbreviated using the notation $\{I\}$. The vector from

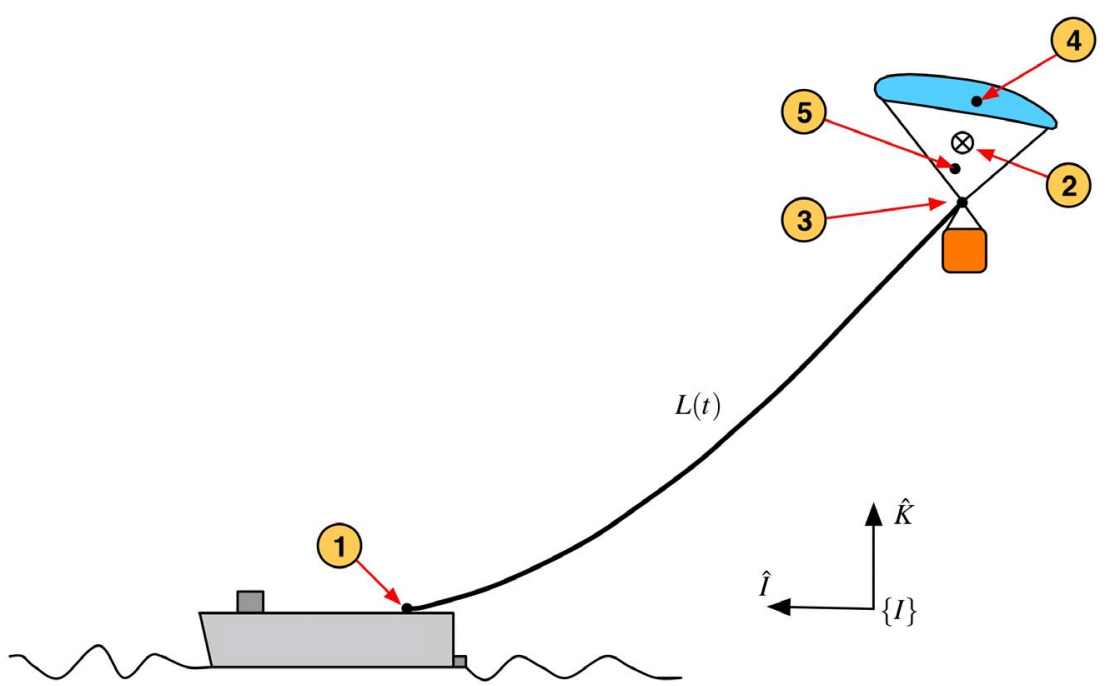

Figure 2. An illustration of the parasail during tow-up. The primary points (1-5) used for developing the dynamic equations are labeled.

the origin of $\{I\}$ to the ship's tow point is denoted as $\vec{p}_{1}(t)$, and is assumed to be known. Thus, the tow point's absolute velocity and acceleration vectors are also known and are denoted as $\vec{v}_{1}(t)$ and $\vec{a}_{1}(t)$ respectively. 


\section{Modeling of the System}

Figure 3 shows a close-up of the parasail/payload assembly to illustrate the body frame, $\{b\}$ used in the dynamic equation development. Its origin is at the center of mass of the parasail/payload system and is oriented relative to $\{I\}$ using a standard yaw, pitch, roll, Euler angle transformation. Thus, the rotation matrix that transforms a vector represented in $\{I\}$ to a representation in $\{b\}$, denoted as ${ }_{I}^{b} \mathbf{R}$ is

$$
{ }_{I}^{b} \mathbf{R}=\left[\begin{array}{ccc}
1 & 0 & 0 \\
0 & \cos \phi & \sin \phi \\
0 & -\sin \phi & \cos \phi
\end{array}\left|\left\lfloor\begin{array}{ccc}
\cos \theta & 0 & -\sin \theta \\
0 & 1 & 0 \\
\sin \theta & 0 & \cos \theta
\end{array}\right]\right| \begin{array}{ccc}
\cos \psi & \sin \psi & 0 \\
-\sin \psi & \cos \psi & 0 \\
0 & 0 & 1
\end{array}\right]
$$

where $\phi, \theta$ and $\psi$ are the roll, pitch, and yaw of the body.

The translational dynamic equations are obtained by applying Newton's second law to the system, considering aerodynamic $\left(\vec{F}_{a}\right)$, gravitational $\left(\vec{F}_{g}\right)$, tow line tension forces $\left(\vec{F}_{t}\right)$. The system free body diagram is shown in Figure 4, and the resulting translational dynamic equation in Eq. 2.

$$
m \vec{a}_{2}=\vec{F}_{g}+\vec{F}_{a}+\vec{F}_{t}+\vec{F}_{e}
$$

where $\vec{a}_{2}$ is the absolute acceleration of the system's center of mass. The gravitational force vector, represented in $\{I\}$ and denoted as ${ }^{I} \vec{F}_{g}$, is

$$
{ }^{I} \vec{F}_{g}=-m g \hat{K}
$$

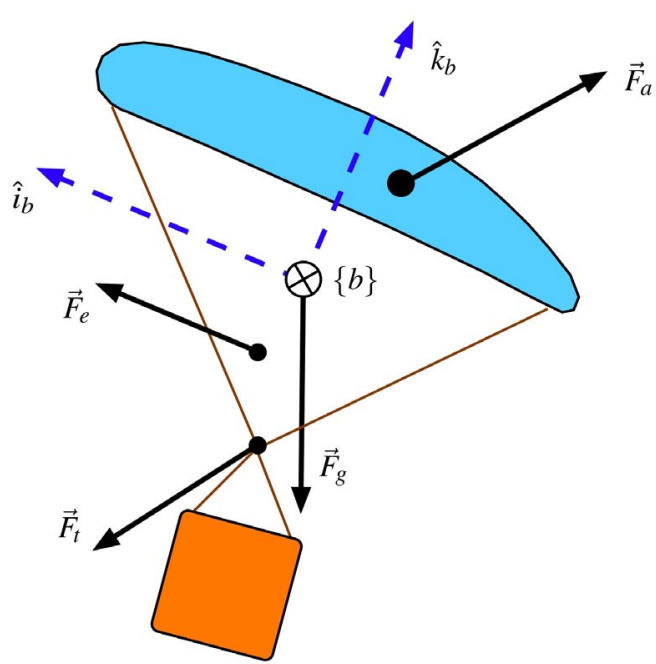

Figure 4. Free body diagram of the parasail/payload system illustrating the 4 external force vectors.

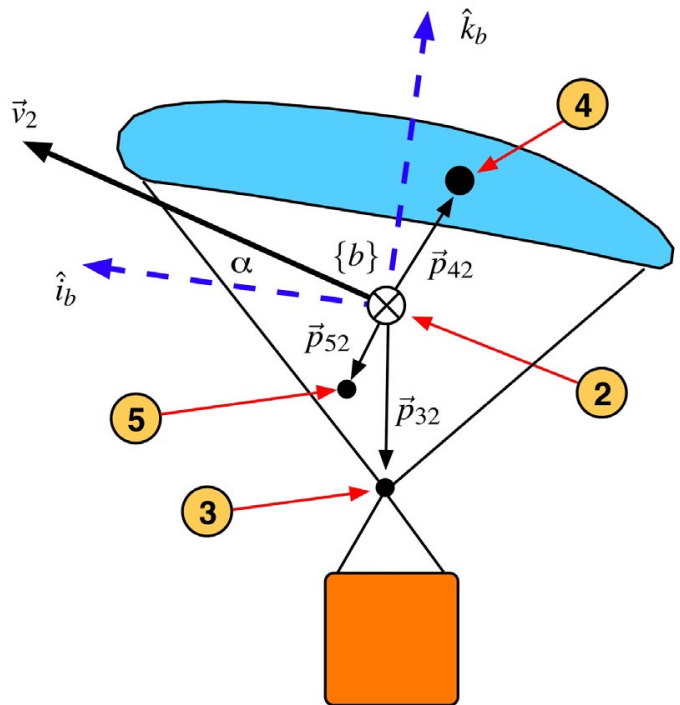

Figure 3. Close-up, side-view of the parasail/payload system showing the body frame, angle of attack $(\alpha)$, and the absolute velocity of the center of mass.

The force due to the constant thrust engine acts along the $\hat{i}_{b}$ direction and is ${ }^{b} \vec{F}_{e}=F_{e} \hat{i}_{b}$ when expressed in $\{b\}$.

The aerodynamic force vector is further decomposed into lift and drag components as shown in Eq. 3.

$\vec{F}_{a}=\vec{F}_{\text {lift }}+\vec{F}_{\text {drag }}$

The magnitude of the lift force is $\left|\vec{F}_{\text {lift }}\right|=c_{L} Q_{L} A$ where $c_{L}$ is the lift aerodynamic coefficient, $Q_{L}$ is the lifting dynamic pressure and $A$ is the effective area of the parasail. Similarly, the magnitude of the drag force is $\left|\vec{F}_{\text {drag }}\right|=c_{D} Q A$ where $c_{D}$ is the aerodynamic drag coefficient and $Q$ is the total dynamic pressure. Since lift and drag are caused by the velocity of the parasail relative to the local wind velocity, the wind relative velocity of the center of pressure point is introduced and defined as 
$\vec{v}_{4, w}=\vec{v}_{2}+\frac{d \vec{r}_{42}}{d t}+\vec{\omega}_{b} \times \vec{r}_{42}-\vec{v}_{w}$

where $\vec{v}_{w}$ is the absolute wind velocity in the vicinity of the parasail and $\vec{\omega}_{b}$ is the absolute angular velocity of $\{b\}$ as shown in Eq. 5 where it is also represented in $\{b\}$.

${ }^{b} \vec{\omega}_{b}=\left[\begin{array}{c}\dot{\phi}-\dot{\psi} \sin \theta \\ \dot{\theta} \cos \phi+\dot{\psi} \cos \theta \sin \phi \\ -\dot{\theta}+\dot{\psi} \cos \theta \cos \phi\end{array}\right]=\Omega\left\{\begin{array}{c}\dot{\phi} \\ \dot{\theta} \\ \dot{\psi}\end{array}\right\}$

(6)

where

$\boldsymbol{\Omega}=\left[\begin{array}{ccc}1 & 0 & -\sin \theta \\ 0 & \cos \phi & \cos \theta \sin \phi \\ 0 & -1 & \cos \theta \cos \phi\end{array} \mid\right.$

Since it is assumed that the parasail is fully inflated, the location of the center of pressure relative to the center of mass is constant. Therefore, $\vec{v}_{4, w}$ can be simplified as

$\vec{v}_{4, w}=\vec{v}_{2}+\vec{\omega}_{b} \times \vec{r}_{42}-\vec{v}_{w}$

The lifting dynamic pressure is assumed to be unaffected by the span-wise contribution of wind speed and is given in Eq. 8.

$Q_{L}=\frac{1}{2} \rho \sqrt{{ }^{b} v_{4 / w, x}^{2}+{ }^{b} v_{4 / w, z}^{2}}$

where $\rho$ is the local density of air and the left superscript indicates components of the vector in $\{b\}$, specifically

${ }^{b} \vec{v}_{4 / w}=\left[\begin{array}{c}{ }^{b} v_{4 / w, x} \\ { }^{b} v_{4 / w, y} \\ { }^{b} v_{4 / w, z}\end{array}\right]$

The total dynamic pressure, used to compute $\vec{F}_{d r a g}$, is simply

$Q=\frac{1}{2} \rho \vec{v}_{4 / w} \cdot \vec{v}_{4 / w}$

Both the lift and drag aerodynamic coefficients are assumed to be linear functions of the angle of attack and are given in Eq. 11. 
$c_{L}=c_{L, 0}+c_{L, \alpha} \alpha$

$c_{D}=c_{D, 0}+c_{D, \alpha} c_{L}^{2}$

where $c_{L, 0}, c_{L, \alpha}, c_{D, 0}$, and $c_{D, \alpha}$ are assumed to be constant and the angle of attack, $\alpha$, is the angle between $\hat{i}_{b}$ and the wind relative velocity vector, $\vec{v}_{4 / w}$, as given by Eq. 12 .

$\alpha=\tan ^{-1}\left(\frac{-v_{4 / w, z}}{v_{4 / w, x}}\right)$

The direction of the lift vector is orthogonal to $\vec{v}_{4 / w}$, and when represented in $\{b\}$, is given by Eq. 13 .

${ }^{b} \vec{F}_{\text {lift }}=\left|F_{\text {lift }}\right|\left[\begin{array}{lll}\sin \alpha & 0 & \cos \alpha\end{array}\right]^{T}$

The direction of drag is opposite to $\vec{v}_{4 / w}$ and so the drag force can be represented as shown in Eq. 14 .

$\vec{F}_{\text {drag }}=-\left|F_{\text {drag }}\right| \hat{v}_{4 / w}$

where $\hat{v}_{4 / w}$ is the unit vector associated with $\vec{v}_{4 / w}$.

Finally, the tow line force is approximated as being along the line connecting the ship and tow point on the parasail system and is described by Eq. 15.

$\vec{F}_{T}=\left|F_{T}\right| \hat{r}_{13}$

where the unit vector $\hat{r}_{13}$ is a function of the ship's position, which is known, and the position of the tow point, which is a function of the parasail's states. This expression is given by Eq. 16 .

$\hat{p}_{13}=\frac{\vec{p}_{1}-\vec{p}_{3}}{\left|\vec{p}_{1}-\vec{p}_{3}\right|}$

The value of $F_{T}$ is an unknown but will be solved at each integration step when the accelerations are computed. The details of this will be described shortly.

The rotational dynamic equations are derived from Euler's equation and the free body diagram of Figure 3 and are given in Eq. 17.

$$
I \dot{\vec{\omega}}+\vec{\omega} \times I \cdot \vec{\omega}=\vec{p}_{32} \times \vec{F}_{T}+\vec{p}_{42} \times \vec{F}_{a}+\vec{p}_{52} \times \vec{F}_{e}
$$

The terms of Eq. 17 have all been introduced above, and thus is completely described.

The 12 states, integrated during the simulation, are given in Eq. 18. 


$$
\vec{x}=\left\{\begin{array}{c}
\phi \\
\theta \\
\psi \\
{ }^{I} \vec{p}_{2} \\
{ }^{b} \vec{\omega}_{b} \\
{ }^{b} \vec{v}_{2}
\end{array}\right\}
$$

At each time step $\dot{\vec{x}}$ must be computed. The time derivative of roll, pitch, and yaw is easily computed using $\boldsymbol{\Omega}$, introduced earlier, and extracting ${ }^{b} \vec{\omega}_{b}$ from the state vector, specifically,

$$
\left\{\begin{array}{l}
\dot{\phi} \\
\dot{\theta} \\
\dot{\psi}
\end{array}\right\}=\Omega^{-1}\left({ }^{b} \vec{\omega}_{b}\right)
$$

The next element of $\dot{\vec{x}},{ }^{I} \vec{v}_{2}$, is readily computed by extracting ${ }^{b} \vec{v}_{2}$ from $\vec{x}$ as shown in Eq. 20.

$$
{ }^{I} \vec{v}_{2}={ }_{I}^{b} \mathbf{R}^{T}\left({ }^{b} \vec{v}_{2}\right)
$$

The angular acceleration, ${ }^{b} \dot{\vec{\omega}}_{b}$ and the time derivative of ${ }^{b} \vec{v}_{2}$ are solved simultaneously with the tow cable force magnitude, $F_{T}$. A set of 7 equations in 7 unknowns (three components of ${ }^{b} \dot{\vec{\omega}}_{b}$, three components of $d\left({ }^{b} \vec{v}_{2}\right) / d t$ and the scalar $F_{t}$ are created in matrix form at each integration time step. by inverting the $7 \times 7$ matrix, the terms in Eq. 18 are computed, as well as the tow cable tension. The tow cable tension is not used in the ensuing integration, but is a useful piece of information from an analysis perspective.

The first 3 equations are created from Eq. 17 where they are written below terms containing solved-for quantities on the left and known terms on right.

$$
I \dot{\vec{\omega}}-\vec{p}_{32} \times \vec{F}_{T}=-\vec{\omega} \times I \cdot \vec{\omega}+\vec{p}_{42} \times \vec{F}_{a}+\vec{p}_{52} \times \vec{F}_{e}
$$

The next 3 equations are created using Eq. 2 and noting that

$$
{ }^{b} \vec{a}_{2}=\frac{d^{b} \vec{v}_{2}}{d t}+{ }^{b} \vec{\omega}_{b} \times{ }^{b} \vec{v}_{2}
$$

Thus, the quantity required for the time derivative of Eq. 18 is

$$
m \frac{d^{b} \vec{v}_{2}}{d t}-F_{T} \hat{p}_{13}=-m^{b} \vec{\omega}_{b} \times{ }^{b} \vec{v}_{2}+{ }^{b} \vec{F}_{a}+{ }^{b} \vec{F}_{g}+{ }^{b} \vec{F}_{e}
$$

The last of the 7 equations is formed by taking two time derivatives of the tow line length constraint equation of Eq. 24

$$
\vec{p}_{13} \cdot \vec{p}_{13}=L^{2}
$$


which gives

$$
\vec{v}_{31} \cdot \vec{p}_{31}=L \dot{L}(t)
$$

and

$$
\vec{a}_{31} \cdot \vec{v}_{31}+\vec{v}_{31} \cdot \vec{v}_{31}=L \ddot{L}+\dot{L}^{2}
$$

Representing all quantities in $\{b\}$, and grouping unknowns on the left and known terms on the left converts the acceleration constraint of Eq. 26 to the last equation of the $7 \times 7$ system and is shown in Eq. 27.

$$
\left.\left[\frac{d\left({ }^{b} \vec{v}_{2}\right)}{d t}+{ }^{b} \dot{\vec{\omega}}_{b} \times{ }^{b} \vec{p}_{32}\right] \cdot{ }^{b} \vec{p}_{31}=\left[-{ }^{b} \vec{\omega}_{b} \times{ }^{b} v_{2}+{ }^{b} a_{1}-{ }^{b} \omega_{b} \times\left({ }^{b} \omega_{b} \times{ }^{b} \vec{p}_{32}\right)\right]\right]^{b} \vec{p}_{31}-{ }^{b} \vec{v}_{31} \cdot{ }^{b} \vec{v}_{31}+L \ddot{L}+\dot{L}^{2}
$$

where

$$
{ }^{b} \vec{v}_{31}=\frac{d\left({ }^{b} \vec{p}_{31}\right)}{d t}+{ }^{b} \vec{\omega}_{b} \times{ }^{b} p_{31}=\frac{d\left({ }^{b} \vec{p}_{3}\right)}{d t}-\frac{d\left({ }^{b} \vec{p}_{1}\right)}{d t}+{ }^{b} \vec{\omega}_{b} \times p_{31}
$$

It should be noted that after the tow line is released, Eq. 27 is omitted from the acceleration solution procedure, and a $6 \times 6$ system is solved. Finally, pitching and rolling moments and increased drag, caused by asserting the parasail brake lines, are applied directly to the simulation as external moments and forces.

\section{Simulation Results}

The simulation was used to obtain a better understanding of the challenges associated with tow-up to 2000 feet. The parasail/payload system needs approximately 8 to 10 minutes to be deployed to 600 meters (1969 feet). With a towing vessel speed of $13 \mathrm{~m} / \mathrm{s}$ ( $25.3 \mathrm{knots})$, the towing vessel needs to cover 6240 to 7800 meters (2.9 to 3.6 miles). Wind conditions may modify these requirements. Shorter deployment times translate into larger towing forces, which are one of the primary limiting factors of deployment time.

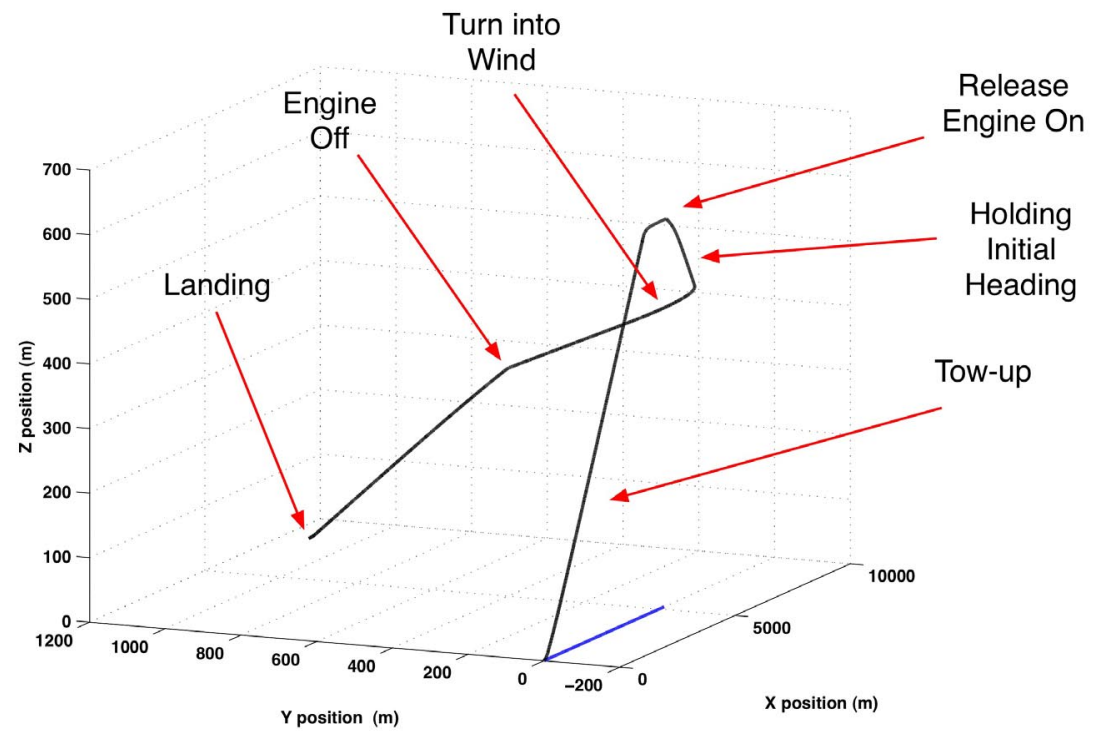

Figure 5. Simulated maneuver evolution from tow-up to landing.

American Institute of Aeronautics and Astronautics 
( 9.8 feet per second) and the towing ship traveling at 13 meters per second ( 25 knots). At 500 seconds, the tow line is released. The engine is turned on at 550 seconds with 400 Newtons of thrust in the body x direction. At 600 seconds the parasail turns into the wind (a 30 degree turn) with engine cut-off at 800 seconds. The landing occurs at approximately 960 seconds into the flight. The landing control strategy is to flare up the pitch angle when the parasail is 4 meters above the ground. This reduces the forward motion by about 2 meters/second, with only a slight gain in downward motion (approximately .3 meters/second). During the flare, the pitch angle goes to approximately 20 degrees. Larger pitch angles are possible, but result in a greater increase in downward speed.

Figure 5 shows the entire evolution in an $\mathrm{x}, \mathrm{y}, \mathrm{z}$ inertial coordinate system. The black line is the parasail trajectory and the blue line is the towing ship. Figure 6 shows the line tension time history, which abruptly ends at the release time. This is kept below $1600 \mathrm{~N}$ using the parasail control system. It is possible that a combination of parasail control and a tension control system on the winch may yield better performance from a time-to-release perspective and robustness. Figure 7 shows the $x-y$ position of the parasail during the flight and Figure 8 shows the altitude time history. These illustrate the change in heading during the evolution and, of course, the change in $\mathrm{z}$ direction.

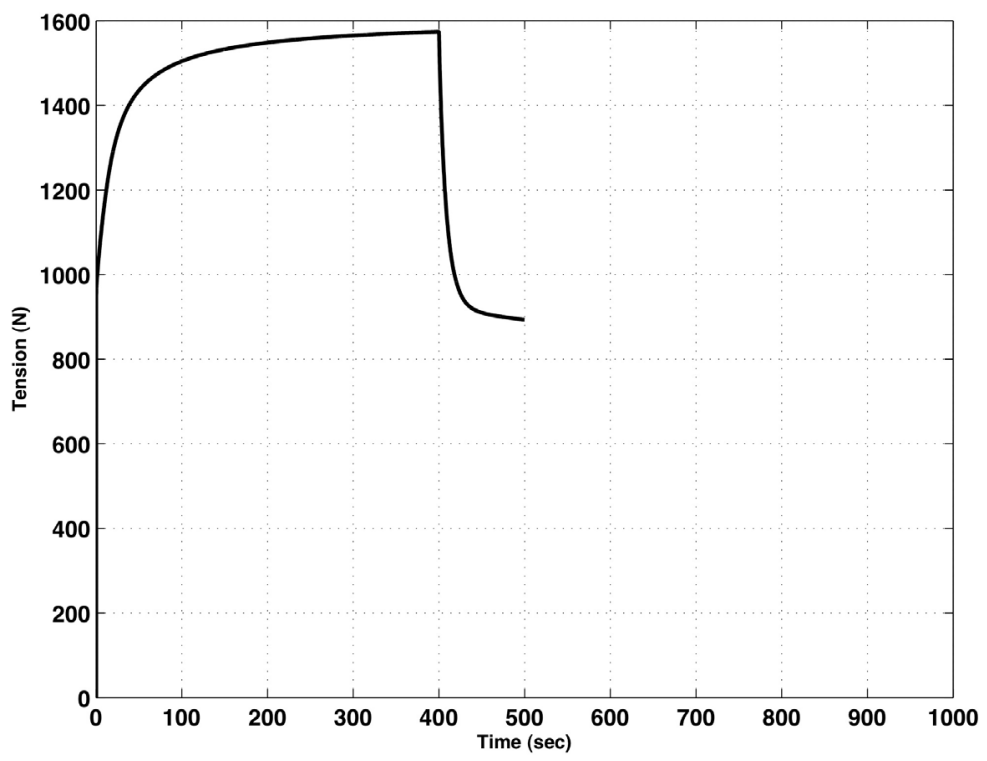

Figure 6. Tow cable tension time history for the maneuver considered in Fig. 5. 


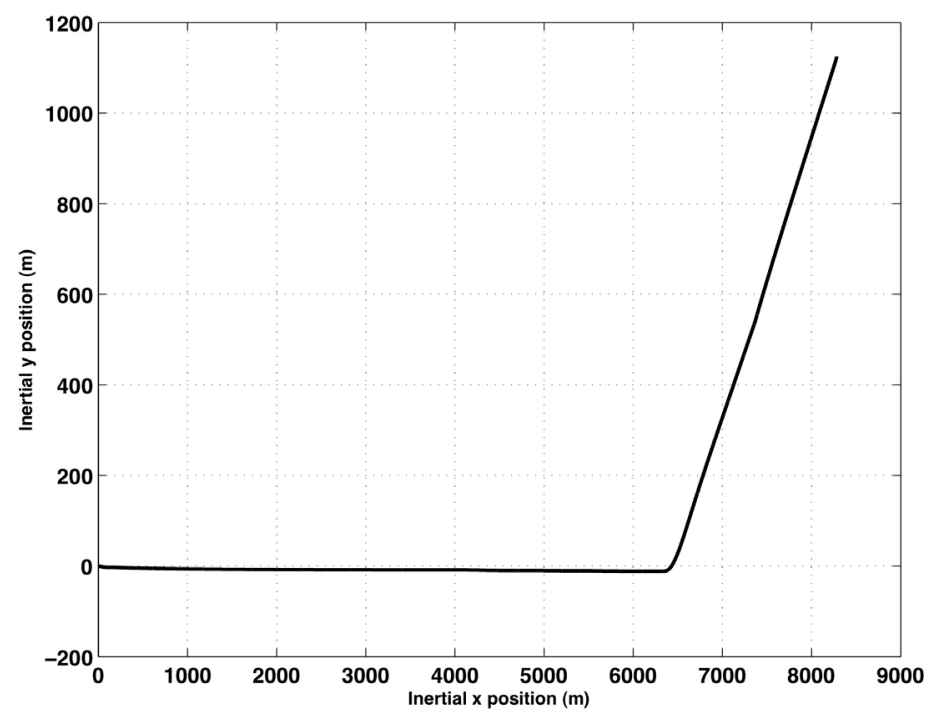

Figure 7. Inertial $\mathrm{x}-\mathrm{y}$ trajectory of the parasail during the maneuver considered in Fig. 5.

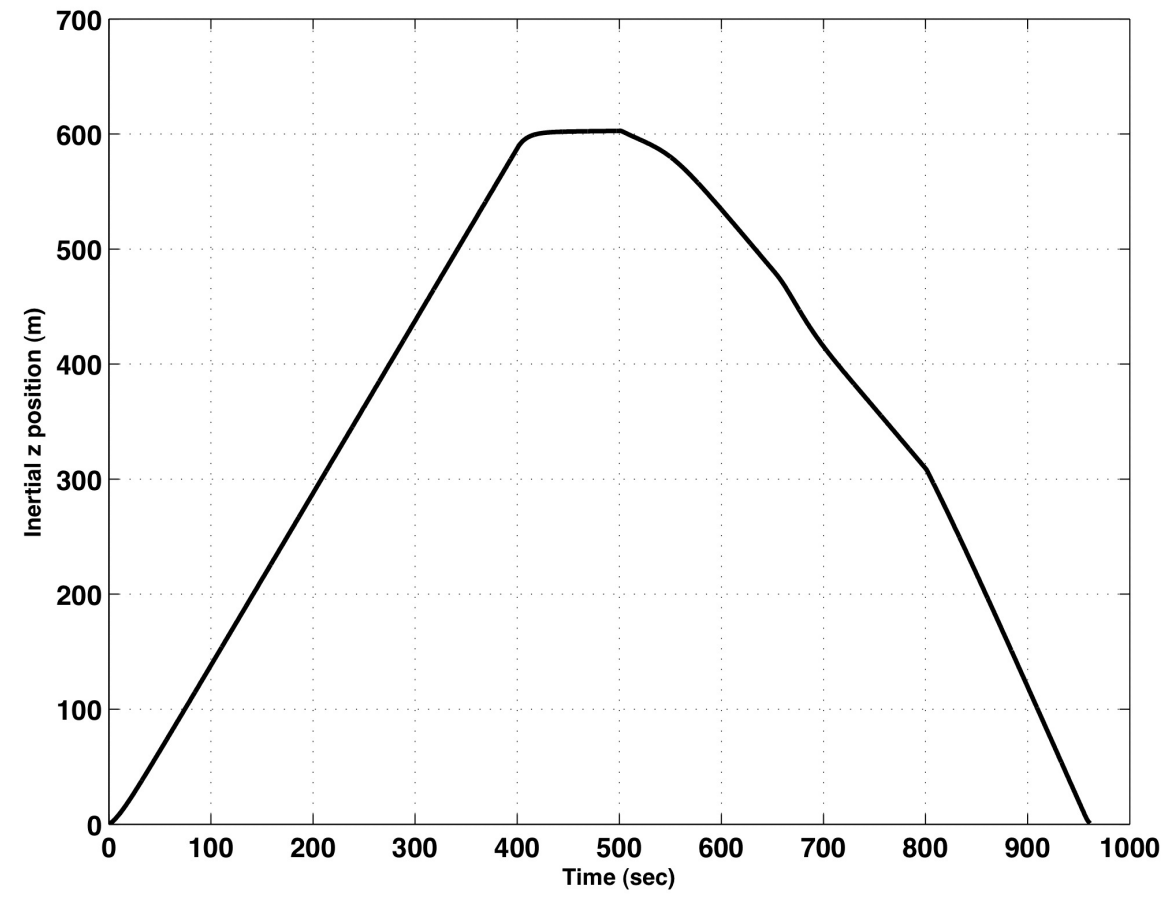

Figure 8. Parasail altitude time history for the maneuver considered in Fig. 5. 


\section{Conclusions}

The complete $6 \mathrm{DoF}$ model of the parafoil system has been developed and realized within the MathWorks MATLAB/Simulink environment. The model demonstrates an adequate response for the control inputs and matches the result of the flight tests well.

An active control strategy is crucial to successful tow-up. Two methods were examined: constant tension with variable tow-line feed rate and attitude/brake control using constant tow-line feed rate. Although both methods appear to work in simulation, it is likely that some combination of the two approaches will be best in practice. One of the primary goals of the control strategy must be to keep the tow-line tension from increasing quickly as this leads to parasail/payload system instability. Furthermore, wind speed sensing will be critical during tow-up and during powered and coast phases of flight. Active control during powered and coast flight, as well as during landing, are extremely important. Not only does this facilitate arriving to a desired location, but also permits wind disturbance rejection

\section{Acknowledgments}

This work was supported by the U.S. Navy under contract number N65538-06-M-0096.

\section{References}

${ }^{1}$ Doherr, K.-F., Jann, T., “Test vehicle ALEX-I for low-cost autonomous parafoil landing experiments,” AIAA Paper 1997-1543, 14 ${ }^{\text {th }}$ AIAA ADST Conference, San Francisco, CA, June 3-5, 1997.

2 Jann, T., "Aerodynamic model identification and GNC design for the parafoil-load system ALEX," AIAA Paper 2001-2015, 16 ${ }^{\text {th }}$ AIAA ADST Conference and Seminar, Boston, MA, May 21-24, 2001.

${ }^{3}$ Mortaloni, P., Yakimenko, O., Dobrokhodov, V., and Howard, R., "On the Development of a Six-Degree-of-Freedom Model of a Low-Aspect-Ratio Parafoil Delivery System," $17^{\text {th }}$ AIAA Aerodynamic Decelerator Systems Technology Conference and Seminar, Monterey, CA, May 19-22, 2003.

4 Barrows, T.M., “Apparent mass of parafoils with spanwise camber,” Journal of Aircraft, 39(3), 2002.

5 Yakimenko, O.A., Statnikov, R.B., "Multicriteria Parametrical Identification of the Parafoil-Load Delivery System," Proc. of the $18^{\text {th }}$ AIAA Aerodynamic Decelerator Systems Technology Conference, Munich, Germany, May 24-26, 2005.

${ }^{6}$ Kaminer, I., Yakimenko, O., "Development of Control Algorithm for the Autonomous Gliding Delivery System," Proc. $17^{\text {th }}$ AIAA Aerodynamic Decelerator Systems Technology Conference and Seminar, Monterey, CA, May 19-22, 2003.

7 Kaminer, I., Pascoal, A.M., Hallberg, E., Silvestre, C., "Trajectory Tracking for Autonomous Vehicles: An Integrated Approach to Guidance and Control," AIAA Journal of Guidance, Control and Dynamics, 21(1), 1998, pp.29-38.

${ }^{8}$ Wolf, D., "Dynamic Stability of Nonrigid Parachute and Payload System," Journal of Aircraft, Vol. 8, No. 8, 1971 , pp 603-609.

9 Müller, S., Wagner, O., Sachs, G., “ A High-Fidelity Nonlinear Multibody Simulation Model For Parafoil Systems,” AIAA paper 2003-2120, $17^{\text {th }}$ Aerodynamic Decelerator Systems Technology Conference and Seminar, Monterey, California, May 19-22, 2003.

${ }^{10}$ Carter, D., George, S., Hattis, P., Singh, L., "Autonomous Guidance, Navigation, and Control of Large Parafoils", AIAA paper 2005-1643, Proc. $18^{\text {th }}$ Aerodynamic Decelerator Systems Technology Conference and Seminar.

${ }^{11}$ Doherr, K., Schilling, H., “ Nine-Degree-of-Freedom Simulation of Rotating Parachute Systems” AIAA paper 91-0877, April 1991.

${ }^{12}$ Slegers, N., Costello, M., “ Aspects of Control for a Parafoil and Payload System”, Journal of Guidance, Control, and Dynamics, Vol.26, No. 6, November-December 2003.

${ }^{13}$ Dobrokhodov, V., Yakimenko, O., Junge, C., "Six-Degree-of-Freedom Model of a Controlled Circular Parachute", Journal of Aircraft, Vol. 40, No. 3, May-June 2003.

${ }^{14}$ Iosilevskii, G., "Center of Gravity and Minimal Lift Coefficient Limits of a Gliding Parachute", Journal of Aircraft, Vol. 32, No. 6, Nov-Dec 1995.

${ }^{15}$ Slegers, N., Costello, M., "Model Predictive Control of A Parafoil and Payload System", AIAA paper 2004-4822. 